\title{
Higgs cross section - an update
}

\author{
V. Ravindran* \\ Harish-Chandra Research Institute, Chhatnag Road, Jhunsi, Allahabad, India \\ E-mail: ravindra@hri.res.in

\section{J. Smith} \\ C.N. Yang Institute for Theoretical Physics, Stony Brook University, Stony Brook, \\ NY 11794-3840 USA \\ E-mail: smith@max2.physics.sunysb.edu
}

\begin{abstract}
We update the Higgs production cross sections at NNLO level and at $\mathrm{N}^{3} \mathrm{LO}$ with only soft-plusvirtual contributions using recent parton density sets. We find that the renormalisation scale dependence is much larger than the factorisation scale dependence in the mass range considered. We also find that the $\mathrm{N}^{3} \mathrm{LO}$ (soft-plus-virtual) corrections reduce the renormalisation scale dependence considerably. We quantify the PDF uncertainty from recent updated PDF sets.
\end{abstract}

10th International Symposium on Radiative Corrections (Applications of Quantum Field Theory to Phenomenology) - Radcor2011

September 26-30, 2011

Mamallapuram, India

${ }^{*}$ Speaker. 
Dedicated studies of high energy physics by both experimentalists and theorists have lead to a better understanding of physics at high scales. The Standard Model (SM) of particle physics, based on the gauge symmetry $S U(3)_{C} \times S U(2)_{L} \times U(1)_{Y}$, has been enormously successful in explaining decades of data from various high energy experiments and other sources. The stringent tests of the SM have been going on for a long time in various high energy facilities and they will continue at the Large Hadron Collider (LHC) at CERN, Geneva. Precise predictions of most of the important observables that can test the SM are now available due to significant advances in theoretical high energy physics. Of course the SM is not expected to explain all of high energy physics and it is known that it has shortcomings. For example, the observed neutrino mass, the dark matter content and the baryon number asymmetry of our universe are a few of the well known examples of observables that the SM has no explanation so far. These issues have been addressed for quite sometime which lead to various extensions of the SM mostly based on enlarged symmetry groups and/or space time dimensions. Like the SM, these extensions have to pass similar tests to establish their relevance to high energy physics. In the electroweak sector of the SM and also in the beyond the SM (BSM) obtaining non-zero masses for the electroweak gauge bosons and the fermionic particles is a hard task because the gauge symmetry that governs the interaction of gauge particles and fermions puts severe restrictions on the their allowed mass terms. The symmetry breaking mechanism plays an important role to achieve this task. In the SM, the weak gauge bosons $W^{ \pm}, Z$ and fermions obtain non-zero masses through the introduction of a complex scalar $S U(2)_{L}$ doublet, called the Higgs field which acquires a non-zero vacuum expectation value. In other words, spontaneous breaking of the $S U(2)_{L} \times U(1)_{Y}$ symmetry to $U(1)_{e m}$ generates masses for weak bosons and fermions. In some of the extensions of SM, similar mechanisms require more than one complex scalar doublet to get the right mass spectrum. This mechanism, often called the Higgs mechanism, is not the only way to achieve this task and there exist several other mechanisms to obtain the required mass spectrum without introducing complex scalar fields. Needless to say all of them have many other predictions which serve as tests for the correctness of these mechanisms.

In the SM the spontaneous symmetry breaking mechanism through a complex scalar doublet not only generates non-zero masses for weak bosons and fermions but also introduces a massive scalar particle, namely the Higgs boson. The Higgs boson has definite couplings to weak bosons and fermions as well as with themselves. The vacuum expectation value of the Higgs field and the mass of the Higgs boson completely determine the scalar sector of the SM. The value of the vacuum expectation value has been known from the observation of weak bosons and their electroweak properties. The only unknown parameter to date is the mass of the Higgs boson, which also determines its coupling to weak bosons and fermions that get their mass through the Higgs mechanism. Direct searches and precision studies of various SM parameters at the LEP and the Tevatron have already provided a wealth of information on where to find the Higgs boson at the LHC. One of the prime goals of the LHC is to look for the the Higgs boson so that the mechanism responsible for the generation of masses for the weak bosons and fermions can be tested. The earlier bounds on the mass reveal that the Higgs boson is not too heavy and it can decay to various SM particles. These decay modes can be used to search for the Higgs boson and also determine its couplings to SM particles. Such an effort has already gone into various studies in earlier experiments and it is underway at the LHC.

Searches for the Higgs boson in collider experiments have been very successful due to dedi- 
cated efforts in searching for observables resulting from various production and decay modes and precise predictions from the theory for these observables. At the LHC Higgs bosons are produced dominantly through gluon-gluon fusion via a top quark loop at leading order in perturbation theory. Due to the non-zero Yukawa coupling for the Higgs boson to top quarks and large gluon flux at the LHC, this dominant production mode can be used in the earlier stages of searches for the Higgs boson. When the energy of the LHC is increased to its designed value and runs with large luminosity over several years then the other subdominant production modes such as vector boson fusion and the associated production of Higgs boson with weak vector bosons and top quarks will be important. In this note, we restrict ourselves to the dominant production mode and the related theoretical developments which lead to a precise prediction for the Higgs production cross section beyond leading order in perturbation theory. This means that the theory of strong interactions, namely Quantum Chromodynamics (QCD) plays an important role in predictions. The Higgs production cross section at the LHC can be computed in QCD using the parton distribution functions $f_{a}\left(w, \mu_{F}^{2}\right)$ of partons in the incoming protons and the parton level scattering cross sections $\Delta_{a b}^{H}$ to produce the Higgs boson:

$$
\sigma^{H}\left(S, m_{H}^{2}\right)=\frac{\pi G_{B}^{2}}{8\left(N^{2}-1\right)} \sum_{a, b=q, \bar{q}, g} \int_{x}^{1} d y \Phi_{a b}\left(y, \mu_{F}^{2}\right) \Delta_{a b}^{H}\left(\frac{x}{y}, m_{H}^{2}, \mu_{F}^{2}, \mu_{R}^{2}\right),
$$

where $x=m_{H}^{2} / S, N=3$ and the factor $G_{B}$ can be found in [1]. The flux $\Phi_{a b}\left(y, \mu_{F}^{2}\right)$ is given by

$$
\Phi_{a b}\left(y, \mu_{F}^{2}\right)=\int_{y}^{1} \frac{d w}{w} f_{a}\left(w, \mu_{F}^{2}\right) f_{b}\left(\frac{y}{w}, \mu_{F}^{2}\right) .
$$

The factorisation and renormalisation scales are given by $\mu_{F}$ and $\mu_{R}$ respectively. The partonic cross section $\Delta_{a b}^{H}$ contains both soft-plus-virtual ( $s v$ ) as well as hard contributions:

$$
\Delta_{a b}^{H}\left(z, m_{H}^{2}, \mu_{R}^{2}, \mu_{F}^{2}\right)=\Delta_{g, S}^{s v}\left(z, m_{H}^{2}, \mu_{R}^{2}, \mu_{F}^{2}\right)+\Delta_{a b}^{H, h a r d}\left(z, m_{H}^{2}, \mu_{R}^{2}, \mu_{F}^{2}\right)
$$

The soft-plus-virtual contributions, as the name suggests, come from all the subprocesses where real gluons in the final states as well as virtual gluons in the loops become soft. The hard part of the partonic cross section is the remaining contribution from these subprocesses.

In perturbative $\mathrm{QCD}$ ( $\mathrm{pQCD}$ ), the partonic cross section for Higgs boson production resulting from the dominant production mechanism, namely gluon-gluon fusion, is known up to next-to-next to leading order (NNLO) level in QCD [2, 3, 4, 5, 6, 7, 8, 9]. Beyond NLO, the Higgs production cross sections are known only in the large top quark mass limit but the corrections due to finite top quark mass are now available in [10]. Two loop electroweak corrections at leading order in $\alpha$ are available, see [11]. One loop corrections to the real radiation processes $q \bar{q} \rightarrow g h$ and $q g \rightarrow q h$ are known, see [12] and for mixed QCD and electroweak contributions with a light quark, see [13]. It is well known that the NLO cross section computed with finite top and bottom quark masses agrees to within rescaled by the $\mathrm{LO}$ order cross section computed with finite top and bottom quark masses even at high Higgs masses. Inclusion of the exact bottom quark loop and its interference with the top quark loop up to NLO level and to resummation upto NLL level can reduce the cross section by about $1.5 \%$ for low Higgs mass. Two loop electroweak corrections can be included either by 
multiplying the full NNLO corrected cross section or by multiplying only LO cross section. In the former case there is an increase in cross section by $5 \%$ while the later gives the variations of the cross section from $-3 \%$ to $+2 \%$ for Higgs mass ranging from 115 to $800 \mathrm{GeV}$. Sub leading terms in the large $m_{t}$ limit give $1 \%$ correction to the NNLO corrected cross section computed in the $m_{t} \rightarrow \infty$ limit for the Higgs mass, $m_{H}<300 \mathrm{GeV}$.

Due to several important results at three loop level that are available $[14,15,16,17,18,19]$, the resummation up to $\mathrm{N}^{3} \mathrm{LL}$ has also become a reality, for resummation exponents see [20, 21, 22, 23]. In [20, 23], the dominant soft gluon contributions to $\mathrm{N}^{3} \mathrm{LO}$ contributions have been computed for the Higgs production cross sections using the form factors and splitting functions that are known up to $\mathrm{N}^{3} \mathrm{LO}$. In [23], this approach has been extended to $\mathrm{N}^{4} \mathrm{LO}$ to get most of the dominant terms that result from soft gluons. In this article, we discuss the impact of these contributions, i.e., soft-plus-virtual contributions at $\mathrm{N}^{3} \mathrm{LO}$ level on the Higgs production cross sections. We will also study the uncertainties resulting from various choices of parton distributions. We do not include electroweak and mixed QCD-electroweak effects in this study because they do not significantly affect the estimates of the error resulting from QCD perturbative results and parton distribution functions. Such effects are well documented in [24, 25] which discusses an update on Higgs cross sections at $8 \mathrm{TeV}$.

Before we present our numerical results, we give a brief overview of how the soft-plus-virtual part of the Higgs cross section is obtained. Thanks to a) factorisation of ultraviolet, soft and collinear contributions in the QCD amplitudes and in the parton level cross sections and b) renormalisation group invariance, we can systematically exponentiate the resulting finite contributions coming from soft gluons present in real and virtual subprocesses after taking into account UV renormalisation and mass factorisation. Note that various UV and infrared singularities cancel among themselves leaving the exponent finite order-by-order in perturbation theory. Hence, the soft-plus-virtual part of the cross section $\left(\Delta_{g, S}^{s v}\left(z, q^{2}, \mu_{R}^{2}, \mu_{F}^{2}\right)\right)$ can be written as

$$
\Delta_{g, S}^{s v}\left(z, q^{2}, \mu_{R}^{2}, \mu_{F}^{2}\right)=\left.\mathscr{C} \exp \left(\Psi_{S}^{g}\left(z, q^{2}, \mu_{R}^{2}, \mu_{F}^{2}, \varepsilon\right)\right)\right|_{\varepsilon=0},
$$

where $\Psi_{S}^{g}\left(z, q^{2}, \mu_{R}^{2}, \mu_{F}^{2}, \varepsilon\right)$ is a finite distribution. Here $\Psi_{S}^{g}\left(z, q^{2}, \mu_{R}^{2}, \mu_{F}^{2}, \varepsilon\right)$ is computed in $4+\varepsilon$ dimensions via

$$
\begin{aligned}
\Psi_{S}^{g}\left(z, q^{2}, \mu_{R}^{2}, \mu_{F}^{2}, \varepsilon\right)= & \left(\ln \left(Z^{g}\left(\hat{a}_{s}, \mu_{R}^{2}, \mu^{2}, \varepsilon\right)\right)^{2}+\ln \left|\hat{F}^{g}\left(\hat{a}_{s}, Q^{2}, \mu^{2}, \varepsilon\right)\right|^{2}\right) \delta(1-z) \\
& +2 \Phi_{S}^{g}\left(\hat{a}_{s}, q^{2}, \mu^{2}, z, \varepsilon\right)-2 \mathscr{C} \ln \Gamma_{g g}\left(\hat{a}_{s}, \mu^{2}, \mu_{F}^{2}, z, \varepsilon\right) .
\end{aligned}
$$

The symbol " $\mathscr{C}$ " means convolution. For example, $\mathscr{C}$ acting on an exponential of a function $f(z)$ has the following expansion:

$$
\mathscr{C} e^{f(z)}=\delta(1-z)+\frac{1}{1 !} f(z)+\frac{1}{2 !} f(z) \otimes f(z)+\frac{1}{3 !} f(z) \otimes f(z) \otimes f(z)+\cdots
$$

In our case $f(z)$ is a distribution such as $\delta(1-z)$ and $\mathscr{D}_{i}$, where

$$
\mathscr{D}_{i}=\left[\frac{\ln ^{i}(1-z)}{(1-z)}\right]_{+} \quad i=0,1, \cdots
$$


and the symbol $\otimes$ is nothing but the Mellin convolution. Here, we drop all the regular functions after all the convolutions are done as we are only interested in the soft-plus-virtual parts of the cross sections.

In the following we explain the various terms that appear in eqn.(5). Since we work in the large top quark mass limit, the top quark degrees of freedom can be safely integrated out. This gives us an effective theory where the Higgs boson can directly couple to the massless gauge bosons, namely the gluons. Because of this an additional renormalisation constant $Z^{g}\left(\hat{a}_{s}, \mu_{R}^{2}, \mu^{2}, \varepsilon\right)$ is required to remove the UV divergences. In the $\overline{\mathrm{MS}}$ scheme, this contains only poles in $\varepsilon$. We have expanded this renormalisation constant in terms of the unrenormalised strong coupling constant $\hat{a}_{s}=\hat{g}_{s}^{2} /\left(16 \pi^{2}\right)$ and the scale $\mu^{2}$ appears in order to keep the coupling constant $\hat{g}_{s}$ dimensionless in $n$ dimensions. $\mu_{R}$ is the UV renormalisation scale at which the parameters and fields of QCD are renormalised. $\hat{F}^{g}\left(\hat{a}_{s}, Q^{2}, \mu^{2}\right)$ is the form factor that describes the Higgs-gluon-gluon vertex expanded in terms of $\hat{a}_{s}$. The scale is given by $Q^{2}=-q^{2}=-m_{H}^{2}$, with $m_{H}$ the mass of the Higgs boson. The scaling variable $z$ is the ratio of $m_{H}^{2} / \hat{s}$, where $\hat{s}$ is the center-of-mass of the partonic system. This function $\Phi_{S}^{g}\left(\hat{a}_{s}, q^{2}, \mu^{2}, z\right)$ is called the soft distribution function. The distribution $\Phi_{S}^{g}\left(\hat{a}_{s}, q^{2}, \mu^{2}, z, \varepsilon\right)$ contains all the soft gluon contributions resulting from parton subprocesses where at least one of the outgoing partons becomes soft. The contributions can come from tree level partonic subprocesses as well as from the virtual processes with at least one or more soft partons in the final state. These can be evaluated again in perturbation theory in terms of $\hat{a}_{s}$.

Both real emission and virtual subprocesses are sensitive to collinear partons which give singular contributions when they are massless. The final state collinear singularities cancel among themselves when they are summed in an infrared safe way. On the other hand, the initial state collinear singularities do not cancel and are removed by mass factorisation. The universal kernel $\Gamma_{g g}\left(\hat{a}_{s}, \mu^{2}, \mu_{F}^{2}, z, \varepsilon\right)$ does this task: it removes the initial state collinear singularities from the partonic subprocesses and renormalises the bare parton distribution functions at the factorisation scale $\mu_{F}$.

The fact that $\Delta_{g, S}^{s v}$ is finite in the limit $\varepsilon \rightarrow 0$ implies that the singular terms in one of the four functions $Z^{g}, \hat{F}^{g}, \Phi_{s}^{g}$ and $\Gamma_{g g}$ can be obtained if the rest are known to sufficiently high orders in $\varepsilon$. While this sounds very simple, in practice one is guided to achieve this by various principles like renormalisation group invariance and factorisation properties of various terms in perturbation theory. Both $\Phi_{S}^{g}$ and $\Gamma_{g g}$ are dependent on the variable $z$ and hence it is not very straightforward to reconstruct the $z$ dependence of one from the others. Note that the pQCD results up to three loop level are known for $Z^{g}, \hat{F}^{g}$ and $\Gamma_{g g}$ but only two loop results are known for $\Phi_{S}^{g}$ from the fixed order computations. Here we extract the three loop QCD contribution with the correct $z$ dependence from $Z^{g}, \hat{F}^{g}$ and $\Gamma_{g g}$ by observing that $\Phi^{g}$ satisfies the following Sudakov type differential equation similar to those for the form factors $\hat{F}^{g}$ :

$$
q^{2} \frac{d}{d q^{2}} \Phi_{S}^{g}\left(\hat{a}_{s}, q^{2}, \mu^{2}, z, \varepsilon\right)=\frac{1}{2}\left[\bar{K}_{S}^{g}\left(\hat{a}_{s}, \frac{\mu_{R}^{2}}{\mu^{2}}, z, \varepsilon\right)+\bar{G}_{H}^{g}\left(\hat{a}_{s}, \frac{q^{2}}{\mu_{R}^{2}}, \frac{\mu_{R}^{2}}{\mu^{2}}, z, \varepsilon\right)\right],
$$

where the constants $\bar{K}_{H}^{g}$ contain all the singular terms and the $\bar{G}_{H}^{g}$ terms are finite functions of $\varepsilon$. We can solve the above equation in powers of $\hat{a}_{s}$ and $\varepsilon$. The particular solution that has the right singular structure and correct $z$ dependence is found to be 


$$
\begin{aligned}
\Phi_{P}^{I}\left(\hat{a}_{s}, q^{2}, \mu^{2}, z, \varepsilon\right) & =\Phi_{P}^{I}\left(\hat{a}_{s}, q^{2}(1-z)^{2}, \mu^{2}, \varepsilon\right) \\
& =\sum_{i=1}^{\infty} \hat{a}_{s}^{i}\left(\frac{q^{2}(1-z)^{2}}{\mu^{2}}\right)^{i \frac{\varepsilon}{2}} S_{\varepsilon}^{i}\left(\frac{i \varepsilon}{1-z}\right) \hat{\phi}_{P}^{I,(i)}(\varepsilon),
\end{aligned}
$$

where

$$
\hat{\phi}_{P}^{I,(i)}(\varepsilon)=\frac{1}{i \varepsilon}\left[\bar{K}^{I,(i)}(\varepsilon)+\bar{G}_{P}^{I,(i)}(\varepsilon)\right]
$$

Note that $\Psi_{S}^{g}$ or equivalently $\Delta_{g, S}^{s v}$ is finite as $\varepsilon \rightarrow 0$ (see eqn.(5)). The constants $\bar{K}^{g,(i)}(\varepsilon)$ are determined by expanding the $\bar{K}_{S}^{g}$ in powers of the bare coupling constant $\hat{a}_{s}$ as

$$
\bar{K}_{S}^{g}\left(\hat{a}_{s}, \frac{\mu_{R}^{2}}{\mu^{2}}, z, \varepsilon\right)=\delta(1-z) \sum_{i=1}^{\infty} \hat{a}_{s}^{i}\left(\frac{\mu_{R}^{2}}{\mu^{2}}\right)^{i \frac{\varepsilon}{2}} S_{\varepsilon}^{i} \bar{K}^{g,(i)}(\varepsilon)
$$

The constants $\bar{G}_{S}^{g,(i)}(\varepsilon)$ are related to the finite function $\bar{G}_{S}^{g}\left(a_{S}\left(q^{2}\right), 1, z, \varepsilon\right)$ through the distributions $\delta(1-z)$ and $\mathscr{D}_{j} . \bar{G}_{S}^{g,(i)}(\varepsilon)$ can be expressed in terms of the renormalised coupling constant $a_{s}$ with the argument $q^{2}(1-z)^{2}$ as

$$
\sum_{i=1}^{\infty} \hat{a}_{S}^{i}\left(\frac{q^{2}(1-z)^{2}}{\mu^{2}}\right)^{i \frac{\varepsilon}{2}} S_{\varepsilon}^{i} \bar{G}_{S}^{g,(i)}(\varepsilon)=\sum_{i=1}^{\infty} a_{S}^{i}\left(q^{2}(1-z)^{2}\right)^{\bar{G}_{S, i}^{g}}(\varepsilon)
$$

The constants $\bar{K}^{g,(i)}(\varepsilon)$ and $\overline{\mathscr{G}}_{S_{i}}^{g}(\varepsilon)$ can be obtained by demanding the finiteness of $\Delta_{g, S}^{s v}$. For example, the $\varepsilon$ dependent terms in $\bar{G}_{S, i}^{g}(\varepsilon)$ are determined from the fixed order (in $a_{s}$ ) computations of cross sections and the finite parts of the form factors. $\overline{\mathscr{G}}_{S, 1}^{g}(\varepsilon)$ is known to all orders in $\varepsilon, \overline{\mathscr{G}}_{S, 2}^{g}(\varepsilon)$ to order $\varepsilon$ and $\overline{\mathscr{G}}_{S, 3}^{g}(\varepsilon)$ to order $\varepsilon^{0}$. The lowest order term $\overline{\mathscr{G}}_{S, 1}^{g}(\varepsilon)$ is known to all orders in $\varepsilon$ because it is often straightforward to compute the fixed order soft contribution at lowest order. On the other hand, it is technically hard to determine $\varepsilon$ dependent parts of soft cross sections beyond the lowest order $a_{s}$. The available constants $\bar{K}^{I,(i)}(\varepsilon)$ and $\overline{\mathscr{G}}_{S_{i}}^{g}(\varepsilon)$ can be found in [26]. Using all this information, the fixed order $\mathrm{N}^{2} \mathrm{LO}$ soft-plus-virtual contribution to Higgs production can be obtained up to terms proportional to $\delta(1-z)$. In other words, we can determine the contributions coming from the distributions $\mathscr{D}^{i}$ at order $\mathrm{N}^{2} \mathrm{LO}$.

In the following, we will present our numerical results for Higgs production at NNLO as well as soft-plus-virtual contributions at $\mathrm{N}^{2} \mathrm{LO}$ in perturbative QCD. We will examine the factorisation and the renormalisation scale dependence of the cross sections and the dependence coming from the choice of parton density sets. We choose the center-of-mass energy to be $\sqrt{S}=8 \mathrm{TeV}$ for the LHC. The standard model parameters that enter our computation are the Fermi constant $G_{F}=$ $4541.68 \mathrm{pb}=1.16637 \times 10^{-5} \mathrm{GeV}^{-2}$ and the top quark mass $m_{t}=172.5 \mathrm{GeV}$. We use LHAPDF to obtain various parton distribution sets and the corresponding strong coupling constants.

In table. 1 we present NNLO and $\mathrm{N}^{3} \mathrm{LO}_{s v}$ corrected Higgs production cross sections for $\sqrt{S}=8$ $\mathrm{TeV}$ using MSTW parton distribution functions [27] and the corresponding $\alpha_{s}$. We present the percentage variation when factorisation and renormalisation scales are independently as well as 


\begin{tabular}{|c|c|c|c|c|c|c|c|c|c|}
\hline$m_{H}$ & NNLO & $\mu_{R}$ & $\mu_{R, 3}$ & $\mu_{F}$ & $\mu$ & $\mathrm{NNLO}_{\bar{\mu}}$ & PDF & $\mathrm{N}^{3} \mathrm{LO}_{s v}$ & $\mu_{R}$ \\
\hline 120 & 19.98 & & & & & 22.07 & $\begin{array}{l}2.49 \\
-3.12\end{array}$ & 20.83 & $\begin{array}{l}+0.12 \\
-2.46\end{array}$ \\
\hline 121 & 19.64 & & & $\begin{array}{l}0.00 \\
-0.58 \\
+0.57\end{array}$ & $\begin{array}{l}+10.46 \\
-9.93\end{array}$ & 21.69 & $\begin{array}{r}+2.49 \\
-3.12\end{array}$ & 20.47 & \\
\hline 122 & 19.31 & $\begin{array}{r}+10.84 \\
-10.43 \\
\end{array}$ & $\begin{array}{l}+16.73 \\
-16.07\end{array}$ & $\begin{array}{l}-0.46 \\
+0.55 \\
\end{array}$ & $\begin{array}{l}+10.45 \\
-9.93\end{array}$ & 21.33 & $\begin{array}{l}+2.50 \\
-3.12\end{array}$ & 20.13 & $\begin{array}{l}+.10 \\
-2.37\end{array}$ \\
\hline 123 & 18.99 & & $\begin{array}{l}+16.70 \\
-16.04\end{array}$ & $\begin{array}{l}-0.43 \\
+0.53\end{array}$ & $\begin{array}{l}+10.44 \\
-9.93\end{array}$ & 20.97 & & 19.79 & $\begin{array}{l}+0.10 \\
-2.32\end{array}$ \\
\hline 124 & 18.68 & & $\begin{array}{l}+16.66 \\
-16.01 \\
\end{array}$ & $\begin{array}{r}-0.41 \\
+0.51 \\
\end{array}$ & $\begin{array}{l}+10.43 \\
-9.94 \\
\end{array}$ & 20.62 & $\begin{array}{l}+2.51 \\
-3.12\end{array}$ & 19.46 & $\begin{array}{r}+0.09 \\
-2.28 \\
\end{array}$ \\
\hline 125 & 18.37 & & + & $\begin{array}{l}0.39 \\
+0.50\end{array}$ & $\begin{array}{l}+1 \\
-9\end{array}$ & 20.28 & $\begin{array}{l}+2.51 \\
-3.13\end{array}$ & 19.13 & $\begin{array}{l}+0.08 \\
-2.24\end{array}$ \\
\hline 126 & 18.07 & & $\begin{array}{l}+16.59 \\
-15.96 \\
\end{array}$ & $\begin{array}{r}-0.37 \\
+0.47\end{array}$ & $\begin{array}{l}+10.42 \\
-9.94\end{array}$ & 19.95 & $\begin{array}{l}+2.52 \\
-3.13\end{array}$ & 18.82 & $\begin{array}{l}+0.07 \\
-2.19\end{array}$ \\
\hline 127 & 17. & & & $\begin{array}{l}-0.35 \\
+0.45\end{array}$ & & 19.6 & $\begin{array}{l}+2.52 \\
-3.13\end{array}$ & 18.51 & $\begin{array}{l}+0.06 \\
-2.15\end{array}$ \\
\hline 128 & 17.49 & $\begin{array}{l}+10.71 \\
-10.31\end{array}$ & $\begin{array}{l}+16.52 \\
-15.91\end{array}$ & $\begin{array}{r}-0.33 \\
+0.42 \\
\end{array}$ & $\begin{array}{l}+10.41 \\
-9.96\end{array}$ & 19.31 & $\begin{array}{l}+2.53 \\
-3.13\end{array}$ & 18.21 & $\begin{array}{l}+0.05 \\
-2.10 \\
\end{array}$ \\
\hline 129 & 17.21 & $\begin{array}{l}-10.31 \\
+10.68 \\
-10.29 \\
\end{array}$ & $\begin{array}{l}-16.41 \\
+15.88 \\
\end{array}$ & $\begin{array}{l}-0.30 \\
+0.34 \\
\end{array}$ & $\begin{array}{l}+10.40 \\
-10.01\end{array}$ & 19.00 & $\begin{array}{l}-2.153 \\
+2.13\end{array}$ & 17.91 & $\frac{-1.10}{+0.04}$ \\
\hline
\end{tabular}

Table 1: $\mathrm{NNLO}$ and $\mathrm{N}^{3} \mathrm{LO}_{s v}$ corrected Higgs production cross sections for $\sqrt{S}=8 \mathrm{TeV}$ as a function of Higgs mass $m_{H}$ using MSTW parton distribution functions. The percentage variations when factorisation and renormalisation scales which are independently as well as simultaneously varied between $m_{H} / 2$ and $2 m_{H}$ and $m_{H} / 3$ and $3 m_{H}$ (denoted by $\mu_{R, 3}$ ) are presented. Those due to the choice of the parton distribution functions within MSTW are also given.

\begin{tabular}{|c|c|c|c|c|c|c|c|c|c|}
\hline$m_{H}$ & NNLO & $\mu_{R}$ & $\mu_{R, 3}$ & $\mu_{F}$ & $\mu$ & $\mathrm{NNLO}_{\bar{\mu}}$ & $\mathrm{PDF}$ & $\mathrm{N}^{3} \mathrm{LO}_{s v}$ & $\mu_{R}$ \\
\hline 120 & 18.51 & $\begin{array}{l}+10.18 \\
-9.91 \\
\end{array}$ & $\begin{array}{l}+15.67 \\
-15.32 \\
\end{array}$ & $\begin{array}{l}-0.24 \\
+0.37 \\
\end{array}$ & $\begin{array}{l}+9.93 \\
-9.63 \\
\end{array}$ & 20.35 & \pm 4.27 & 21.04 & $\begin{array}{l}+0.06 \\
-2.39 \\
\end{array}$ \\
\hline 121 & 18.18 & $\begin{array}{l}10.13 \\
-9.88 \\
\end{array}$ & $\begin{array}{l}+15.58 \\
-15.28 \\
\end{array}$ & $\begin{array}{l}-0.10 \\
+0.40 \\
\end{array}$ & $\begin{array}{l}+10.05 \\
-9.58 \\
\end{array}$ & 20.00 & \pm 4.25 & 20.62 & $\begin{array}{l}+0.17 \\
-2.28 \\
\end{array}$ \\
\hline 122 & 17.89 & $\begin{array}{l}10.15 \\
-9.88 \\
\end{array}$ & $\begin{array}{l}+15.62 \\
-15.28 \\
\end{array}$ & $\begin{array}{l}-0.20 \\
+0.34 \\
\end{array}$ & $\begin{array}{r}+9.91 \\
-9.63 \\
\end{array}$ & 19.66 & \pm 4.35 & 20.32 & $\begin{array}{l}+0.09 \\
-2.29 \\
\end{array}$ \\
\hline 123 & 17.58 & $\begin{array}{l}+10.13 \\
-9.86 \\
\end{array}$ & $\begin{array}{l}+15.59 \\
-15.25 \\
\end{array}$ & $\begin{array}{r}-0.18 \\
+0.33 \\
\end{array}$ & $\begin{array}{r}+9.91 \\
-9.63 \\
\end{array}$ & 19.33 & \pm 4.40 & 19.97 & $\begin{array}{l}+0.10 \\
-2.25 \\
\end{array}$ \\
\hline 124 & 17.29 & $\begin{array}{l}+10.11 \\
-9.85 \\
\end{array}$ & $\begin{array}{l}+15.57 \\
-15.23 \\
\end{array}$ & $\begin{array}{r}-0.16 \\
+0.31 \\
\end{array}$ & $\begin{array}{l}+9.90 \\
-9.64 \\
\end{array}$ & 19.00 & \pm 4.43 & 19.63 & $\begin{array}{l}+0.09 \\
-2.21 \\
\end{array}$ \\
\hline 125 & 16.99 & $\begin{array}{l}+10.08 \\
-9.83 \\
\end{array}$ & $\begin{array}{l}+15.52 \\
-15.20 \\
\end{array}$ & $\begin{array}{l}-0.12 \\
+0.28 \\
\end{array}$ & $\begin{array}{r}+9.91 \\
-9.65 \\
\end{array}$ & 18.67 & \pm 4.75 & 19.28 & $\begin{array}{l}+0.12 \\
-2.15 \\
\end{array}$ \\
\hline 126 & 16.71 & $\begin{array}{l}+10.06 \\
-9.81 \\
\end{array}$ & $\begin{array}{l}+15.49 \\
-15.17 \\
\end{array}$ & $\begin{array}{l}-0.13 \\
+0.25 \\
\end{array}$ & $\begin{array}{r}+9.87 \\
-9.66 \\
\end{array}$ & 18.36 & \pm 4.54 & 18.96 & $\begin{array}{l}+0.13 \\
-2.11 \\
\end{array}$ \\
\hline 127 & 16.43 & $\begin{array}{l}+10.04 \\
-9.79 \\
\end{array}$ & $\begin{array}{l}+15.46 \\
-15.15 \\
\end{array}$ & $\begin{array}{l}-0.03 \\
+0.27 \\
\end{array}$ & $\begin{array}{r}+9.96 \\
-9.63 \\
\end{array}$ & 18.07 & \pm 4.26 & 18.64 & $\begin{array}{l}+0.14 \\
-2.06 \\
\end{array}$ \\
\hline 128 & 16.16 & $\begin{array}{l}+10.01 \\
-9.77 \\
\end{array}$ & $\begin{array}{l}+15.41 \\
-15.12 \\
\end{array}$ & $\begin{array}{l}-0.01 \\
+0.26 \\
\end{array}$ & $\begin{array}{r}+9.99 \\
-9.63 \\
\end{array}$ & 17.77 & \pm 4.69 & 18.32 & $\begin{array}{l}+0.14 \\
-2.02 \\
\end{array}$ \\
\hline 129 & 15.91 & $\begin{array}{l}+10.02 \\
-9.77 \\
\end{array}$ & $\begin{array}{l}+15.42 \\
-15.11 \\
\end{array}$ & $\begin{array}{l}-0.08 \\
+0.05 \\
\end{array}$ & $\begin{array}{l}+9.85 \\
-9.79 \\
\end{array}$ & 17.48 & \pm 4.62 & 18.04 & $\begin{array}{l}+0.15 \\
-1.98 \\
\end{array}$ \\
\hline
\end{tabular}

Table 2: Same as table.1 but with ABM parton distribution functions

simultaneously varied between $m_{H} / 2$ and $2 m_{H}$ (named by $\mu_{R}, \mu_{F}, \mu$ respectively). The column denoted by $\mu_{R, 3}$ is obtained by varying the renormalisation scale in the range $m_{H} / 3 \leq \mu_{R} \leq 3 m_{H}$. In the column denoted by $\mathrm{NNLO}_{\bar{\mu}}$, the cross sections are obtained for a fixed scale $\mu_{R}=\mu_{F}=m_{H} / 2$ which is supposed to mimic the NNLL threshold resummed results. Also we have presented the percentage variation of the cross sections due to the choice of the parton distribution functions within MSTW (denoted by PDF). In the last column, we have listed the percentage variation of the cross section when the renormalisation scale is in the range $m_{H} / 2 \leq \mu_{R} \leq 2 m_{H}$. For $\mathrm{N}^{3} \mathrm{LO}_{s v}$, we have used NNLO PDFs but four loop corrected $\alpha_{s}$ because $\mathrm{N}^{3} \mathrm{LO}_{s v}$ PDFs are not yet available. This approximation is still okay because only mild $\mu_{F}$ dependence is seen at NNLO level. In table. 2 we present similar results using ABM11 parton distribution functions [28]. We find that 


\begin{tabular}{|c|c|c|c|c|c|c|c|c|}
\hline \multirow{2}{*}{$m_{H}$} & \multicolumn{5}{|c|}{ NNLO } & \multicolumn{4}{c|}{$\mathrm{N}^{3} \mathrm{LO}_{s v}$} \\
\cline { 2 - 10 } & MSTW & ABM & CT & NNPDF & MSTW & ABM & CT & NNPDF \\
\hline \hline 120 & 19.98 & 18.51 & 19.86 & 21.00 & 20.83 & 21.04 & 20.26 & 20.91 \\
\hline 121 & 19.64 & 18.18 & 19.52 & 20.65 & 20.47 & 20.62 & 19.91 & 20.56 \\
\hline 122 & 19.31 & 17.89 & 19.20 & 20.30 & 20.13 & 20.32 & 19.57 & 20.21 \\
\hline 123 & 18.99 & 17.58 & 18.88 & 19.96 & 19.79 & 19.97 & 19.24 & 19.87 \\
\hline 124 & 18.68 & 17.29 & 18.57 & 19.63 & 19.46 & 19.63 & 18.92 & 19.54 \\
\hline 125 & 18.37 & 16.99 & 18.27 & 19.31 & 19.13 & 19.28 & 18.61 & 19.21 \\
\hline 126 & 18.07 & 16.71 & 17.97 & 18.99 & 18.82 & 18.96 & 18.31 & 18.89 \\
\hline 127 & 17.78 & 16.43 & 17.68 & 18.66 & 18.51 & 18.64 & 18.01 & 18.53 \\
\hline 128 & 17.49 & 16.16 & 17.39 & 18.52 & 18.21 & 18.32 & 17.72 & 18.61 \\
\hline 129 & 17.21 & 15.91 & 17.12 & 18.09 & 17.91 & 18.04 & 17.43 & 17.99 \\
\hline \hline
\end{tabular}

Table 3: Higgs production cross section as a function of $m_{H}$ using MSTW,ABM, CT and NNPDF parton distribution functions (in particular their central pdfs).

the renormalisation scale gives $10 \%$ variation if $\mu_{R}$ is varied in the range $m_{H} / 2 \leq \mu_{R} \leq 2 m_{H}$ and increases to $16 \%$ in the range $m_{H} / 3 \leq \mu_{R} \leq 3 m_{H}$ indicating that the NNLO corrected cross section does not sufficiently stabilise the cross section warrenting a full $\mathrm{N}^{3} \mathrm{LO}$ corrected results. As is clear from the last column, the $\mathrm{N}^{3} \mathrm{LO}_{s v}$ corrected results show significant reduction in $\mu_{R}$ dependence. In table.3 we present NNLO and $\mathrm{N}^{3} \mathrm{LO}_{s v}$ corrected Higgs production cross sections using CT10 [29] and NNPDF [30] along with results obtained with MSTW and ABM PDFs to compare the results from various PDF groups. We have chosen $\mu_{R}=\mu_{F}=m_{H}$. With respect to the MSTW predictions, $\mathrm{ABM}$ gives $7.5 \%$ smaller cross sections, $\mathrm{CT}$ gives $0.5 \%$ smaller cross sections and NNPDF gives $5 \%$ larger cross sections. At $\mathrm{N}^{3} \mathrm{LO}_{s v}$ level, we find that $\mathrm{ABM}$ give $0.8 \%$ larger cross sections while CT gives $2.7 \%$ smaller cross sections and NNPDF gives $0.41 \%$ larger cross sections. Surprisingly they are all very close to each other over the entire mass range considered here.

In this article, we have updated the Higgs production cross sections at NNLO level computed in the large top mass limit using recent parton density sets. We have also studied the role of $\mathrm{N}^{3} \mathrm{LO}$ soft-plus-virtual effects to reduce the renormalisation scale uncertainty. We find that the renormalisation scale dependence is much larger than the factorisation scale dependence at NNLO level and the $\mathrm{N}^{3} \mathrm{LO}$ (soft-plus-virtual) corrections reduce the renormalisation scale dependence considerably. We also find that the PDF uncertainty is significant not only within a given PDF set, but also among various sets.

Acknowledgement: We thank S. Alekhin and J. Blümlein for their help on ABM PDF sets.

\section{References}

[1] K. G. Chetyrkin, B. A. Kniehl and M. Steinhauser, Nucl. Phys. B 510, 61 (1998) [arXiv:hep-ph/9708255].

[2] S. Dawson, Nucl. Phys. B 359, 283 (1991).

[3] A. Djouadi, M. Spira and P. M. Zerwas, Phys. Lett. B 264, 440 (1991). 
[4] M. Spira, A. Djouadi, D. Graudenz and P. M. Zerwas, Nucl. Phys. B 453, 17 (1995) [arXiv:hep-ph/9504378].

[5] R. V. Harlander and W. B. Kilgore, Phys. Rev. D 64, 013015 (2001) [arXiv:hep-ph/0102241].

[6] S. Catani, D. de Florian and M. Grazzini, JHEP 0105, 025 (2001) [arXiv:hep-ph/0102227].

[7] R. V. Harlander and W. B. Kilgore, Phys. Rev. Lett. 88, 201801 (2002) [arXiv:hep-ph/0201206].

[8] C. Anastasiou and K. Melnikov, Nucl. Phys. B 646, 220 (2002) [arXiv:hep-ph/0207004].

[9] V. Ravindran, J. Smith and W. L. van Neerven, Nucl. Phys. B 665, 325 (2003) [arXiv:hep-ph/0302135].

[10] R. V. Harlander and K. J. Ozeren, JHEP 0911 (2009) 088, [arXiv:0909.3420 [hep-ph]]. A. Pak, M. Rogal and M. Steinhauser, JHEP 1002 (2010) 025, [arXiv:0911.4662 [hep-ph]].

[11] S. Actis, G. Passarino, C. Sturm and S. Uccirati, Nucl. Phys. B 811, 182 (2009) [arXiv:ihep-ph/0809.3667] S. Actis, G. Passarino, C. Sturm and S. Uccirati, Phys. Lett. B 670, 12 (2008) [arXiv:hep-ph/0809.1301]. U. Aglietti, R. Bonciani, G. Degrassi and A. Vicini, Phys. Lett. B 595, 432 (2004) [arXiv:hep-ph/0404071].

[12] W. Y. Keung and F. J. Petriello, Phys. Rev. D 80, 013007 (2009) [arXiv:hep-ph/0905.2775].

[13] C. Anastasiou, R. Boughezal and F. Petriello, JHEP 0904, 003 (2009) [arXiv:hep-ph/0811.3458].

[14] S. Moch, J. A. M. Vermaseren and A. Vogt, Nucl. Phys. B 688, 101 (2004) [arXiv:hep-ph/0403192].

[15] A. Vogt, S. Moch and J. A. M. Vermaseren, Nucl. Phys. B 691, 129 (2004) [arXiv:hep-ph/0404111].

[16] S. Moch, J. A. M. Vermaseren and A. Vogt, JHEP 0508, 049 (2005) [arXiv:hep-ph/0507039].

[17] S. Moch, J. A. M. Vermaseren and A. Vogt, Phys. Lett. B 625, 245 (2005) [arXiv:hep-ph/0508055].

[18] S. Moch, J. A. M. Vermaseren and A. Vogt, Nucl. Phys. B 726, 317 (2005) [arXiv:hep-ph/0506288].

[19] J. Blũmlein and J. A. M. Vermaseren, Phys. Lett. B 606, 130 (2005) [arXiv:hep-ph/0411111].

[20] S. Moch and A. Vogt, Phys. Lett. B 631, 48 (2005) [arXiv:hep-ph/0508265].

[21] E. Laenen and L. Magnea, Phys. Lett. B 632, 270 (2006) [arXiv:hep-ph/0508284].

[22] A. Idilbi, X. d. Ji, J. P. Ma and F. Yuan, Phys. Rev. D73 (2006) 077501 [arXiv:hep-ph/0509294].

[23] V. Ravindran, Nucl. Phys. B 746 (2006) 58 [arXiv:hep-ph/0512249].

[24] D. de Florian, M. Grazzini and M. Grazzini, arXiv:1206.4133 [hep-ph].

[25] C. Anastasiou, S. Buehler, F. Herzog and A. Lazopoulos, JHEP 1204 (2012) 004 [arXiv:1202.3638 [hep-ph]].

[26] V. Ravindran, Nucl. Phys. B 752 (2006) 173 [arXiv:hep-ph/0603041].

[27] A. D. Martin, W. J. Stirling, R. S. Thorne and G. Watt, Eur. Phys. J. C 63 (2009) 189 [arXiv:hep-ph/0901.0002].

[28] S. Alekhin, J. Blumlein and S. Moch, Phys. Rev. D 86 (2012) 054009 [arXiv:hep-ph/1202.2281].

[29] H. -L. Lai, M. Guzzi, J. Huston, Z. Li, P. M. Nadolsky, J. Pumplin and C. -P. Yuan, Phys. Rev. D 82 (2010) 074024 [arXiv:hep-ph/1007.2241].

[30] R. D. Ball et al. [NNPDF Collaboration], Nucl. Phys. B 855 (2012) 153 [arXiv:hep-ph/1107.2652]. 